\title{
Some new integral inequalities for n-times differentiable convex and concave functions
}

Selahattin Maden $^{\mathrm{a}}$, Huriye Kadakal ${ }^{\mathrm{b}}$, Mahir Kadakal ${ }^{\mathrm{c}, *}$, Immdat İşcan ${ }^{\mathrm{c}}$

${ }^{a}$ Department of Mathematics, Faculty of Sciences and Arts, Ordu University-Ordu-TÜRKIYE.

${ }^{b}$ Institute of Science, Ordu University-Ordu-TÜRKIYE.

${ }^{c}$ Department of Mathematics, Faculty of Sciences and Arts, Giresun University-Giresun-TÜRKIYE.

Communicated by M. Bohner

\begin{abstract}
In this work, by using an integral identity together with both the Hölder and the Power-mean integral inequalities we establish several new inequalities for n-times differentiable convex and concave mappings. (C)2017 All rights reserved.
\end{abstract}

Keywords: Convex function, concave function, Hölder integral inequality, power-mean integral inequality. 2010 MSC: 26A51, 26D10, 26D15.

\section{Introduction}

Let $f: I \subseteq \mathbb{R} \rightarrow \mathbb{R}$ be a concave function on the interval I of real numbers and $a, b \in$ I with $a<b$. The inequality

$$
\frac{f(a)+f(b)}{2} \leqslant \frac{1}{b-a} \int_{a}^{b} f(x) d x \leqslant f\left(\frac{a+b}{2}\right),
$$

is well-known in the literature as Hermite-Hadamard's inequality for concave functions [16]. Both inequalities hold in the reserved direction if $f$ is convex. The classical Hermite-Hadamard inequality provides estimates of the mean value of a continuous convex or concave function. Hadamard's inequality for convex or concave functions has received renewed attention in recent years and a remarkable variety of refinements and generalizations have been found; for example see [1-16].

A function $f: I \subseteq \mathbb{R} \rightarrow \mathbb{R}$ is said to be convex if the inequality

$$
f(t x+(1-t) y) \leqslant t f(x)+(1-t) f(y),
$$

is valid for all $x, y \in I$ and $t \in[0,1]$. If this inequality reverses, then $f$ is said to be concave on interval

\footnotetext{
*Corresponding author

Email addresses: maden55@mynet.com (Selahattin Maden), huriyekadakal@hotmail.com (Huriye Kadakal), mahirkadakal@gmail . com (Mahir Kadakal), imdat.iscan@giresun.edu .tr (İmdat İşcan)

doi:10.22436/jnsa.010.12.01
} 
$\mathrm{I} \neq \varnothing$. This definition is well-known in the literature. Convexity theory has appeared as a powerful technique to study a wide class of unrelated problems in pure and applied sciences. For some inequalities, generalizations and applications concerning convexity see [3, 5-11, 15-18]. Recently, in the literature there are so many papers about $n$-times differentiable functions on several kinds of convexities. In references $[2-4,6,13,17,19]$, readers can find some results about this issue. Many papers have been written by a number of mathematicians concerning inequalities for different classes of convex functions see for instance the recent papers $[1,5,7-12,14]$ and the references within these papers.

Let $0<a<b$, throughout this paper we will use

$$
\begin{aligned}
A(a, b) & =\frac{a+b}{2} \\
G(a, b) & =\sqrt{a b} \\
L_{a}(a, b) & =\left(\frac{b^{p+1}-a^{p+1}}{(p+1)(b-a)}\right)^{\frac{1}{p}}, \quad a \neq b, \quad p \in \mathbb{R}, \quad p \neq-1,0,
\end{aligned}
$$

for the arithmetic, geometric, generalized logarithmic means, respectively.

\section{Main results}

We will use the following for obtain our main results.

Lemma 2.1. Let $\mathrm{f}: \mathrm{I} \subseteq \mathbb{R} \rightarrow \mathbb{R}$ be $\mathrm{n}$-times differentiable mapping on $\mathrm{I}^{\circ}$ for $\mathrm{n} \in \mathbb{N}$ and $\mathrm{f}^{(\mathrm{n})} \in \mathrm{L}[\mathrm{a}, \mathrm{b}]$, where $\mathrm{a}, \mathrm{b} \in \mathrm{I}^{\circ}$ with $\mathrm{a}<\mathrm{b}$. We have the identity

$$
\sum_{k=0}^{n-1}(-1)^{k}\left(\frac{f^{(k)}(b) b^{k+1}-f^{(k)}(a) a^{k+1}}{(k+1) !}\right)-\int_{a}^{b} f(x) d x=\frac{(-1)^{n+1}}{n !} \int_{a}^{b} x^{n} f^{(n)}(x) d x,
$$

where an empty sum is understood to be nil.

Proof. To prove, we shall use the induction method. For $n=1$, by integration by parts we have

$$
f(b) b-f(a) a-\int_{a}^{b} f(x) d x=\int_{a}^{b} x f^{\prime}(x) d x .
$$

This coincides with the equality (2.1) for $n=1$. Similarly for $n=2$ and using integration by parts as above, we have

$$
\begin{aligned}
& \sum_{k=0}^{1}(-1)^{k}\left(\frac{f^{(k)}(b) b^{k+1}-f^{(k)}(a) a^{k+1}}{(k+1) !}\right)-\int_{a}^{b} f(x) d x=\frac{(-1)^{2+1}}{2 !} \int_{a}^{b} x^{2} f^{\prime \prime}(x) d x, \\
& \frac{f(b) b-f(a) a}{1 !}-\frac{f^{\prime}(b) b^{2}-f^{\prime}(a) a^{2}}{2 !}-\int_{a}^{b} f(x) d x=-\frac{1}{2 !} \int_{a}^{b} x^{2} f^{\prime \prime}(x) d x, \\
& \int_{a}^{b} x^{2} f^{\prime \prime}(x) d x=f^{\prime}(b) b^{2}-f^{\prime}(a) a^{2}-2\left[\left.x f(x)\right|_{a} ^{b}-\int_{a}^{b} f(x) d x\right] \\
& =f^{\prime}(b) b^{2}-f^{\prime}(a) a^{2}-2[b f(b)-a f(a)]+2 \int_{a}^{b} f(x) d x .
\end{aligned}
$$

Equation (2.2) coincides with the equality (2.1) for $n=2$.

Suppose (2.1) holds for $\mathrm{n}=\mathrm{t}$. That is

$$
\sum_{k=0}^{t-1}(-1)^{k}\left(\frac{f^{(k)}(b) b^{k+1}-f^{(k)}(a) a^{k+1}}{(k+1) !}\right)-\int_{a}^{b} f(x) d x=\frac{(-1)^{t+1}}{t !} \int_{a}^{b} x^{t} f^{(t)}(x) d x .
$$


Using the integration by parts we have

$$
\begin{aligned}
\frac{(-1)^{t+2}}{(t+1) !} \int_{a}^{b} x^{t+1} f^{(t+1)}(x) d x & =\frac{(-1)^{t+2}}{(t+1) !}\left\{\left.x^{t+1} f^{(t)}(x)\right|_{a} ^{b}-(t+1) \int_{a}^{b} x^{t} f^{(t)}(x) d x\right\} \\
& =\frac{(-1)^{t}}{(t+1) !}\left[f^{(t)}(b) b^{t+1}-f^{(t)}(a) a^{t+1}\right]-(t+1) \frac{(-1)^{t+2}}{(t+1) !} \int_{a}^{b} x^{t} f^{(t)}(x) d x \\
& =\frac{(-1)^{t+2}}{(t+1) !}\left[f^{(t)}(b) b^{t+1}-f^{(t)}(a) a^{t+1}\right]+\frac{(-1)^{t+1}}{t !} \int_{a}^{b} x^{t} f^{(t)}(x) d x
\end{aligned}
$$

Substituting (2.4) in (2.3) we obtain

$$
\begin{array}{r}
\sum_{k=0}^{t-1}(-1)^{k}\left(\frac{f^{(k)}(b) b^{k+1}-f^{(k)}(a) a^{k+1}}{(k+1) !}\right)-\int_{a}^{b} f(x) d x=\frac{(-1)^{t+1}}{t !} \int_{a}^{b} x^{t} f^{(t)}(x) d x, \\
\begin{aligned}
\sum_{k=0}^{t-1}(-1)^{k}\left(\frac{f^{(k)}(b) b^{k+1}-f^{(k)}(a) a^{k+1}}{(k+1) !}\right)-\int_{a}^{b} f(x) d x= & \frac{(-1)^{t+2}}{(t+1) !} \int_{a}^{b} x^{t+1} f^{(t+1)}(x) d x \\
& -\frac{(-1)^{t}}{(t+1) !}\left[f^{(t)}(b) b^{t+1}-f^{(t)}(a) a^{t+1}\right],
\end{aligned}
\end{array}
$$

that is,

$$
\begin{aligned}
& \sum_{k=0}^{t-1}(-1)^{k}\left(\frac{f^{(k)}(b) b^{k+1}-f^{(k)}(a) a^{k+1}}{(k+1) !}\right)-\int_{a}^{b} f(x) d x+\frac{(-1)^{t}}{(t+1) !}\left[f^{(t)}(b) b^{t+1}-f^{(t)}(a) a^{t+1}\right] \\
& =\frac{(-1)^{t+2}}{(t+1) !} \int_{a}^{b} x^{t+1} f^{(t+1)}(x) d x .
\end{aligned}
$$

This completes the proof of Lemma.

Theorem 2.2. For $\mathrm{n} \in \mathbb{N}$, let $\mathrm{f}: \mathrm{I} \subseteq(0, \infty) \rightarrow \mathbb{R}$ be $\mathrm{n}$-times differentiable function on $\mathrm{I}^{\circ}$ and $\mathrm{a}, \mathrm{b} \in \mathrm{I}^{\circ}$ with $\mathrm{a}<\mathrm{b}$. If $\mathrm{f}^{(\mathrm{n})} \in \mathrm{L}[\mathrm{a}, \mathrm{b}]$ and $\left|\mathrm{f}^{(\mathrm{n})}\right|^{\mathrm{q}}$ for $\mathrm{q}>1$ is convex on $[\mathrm{a}, \mathrm{b}]$, then the following inequality holds:

$$
\begin{aligned}
& \left|\sum_{k=0}^{n-1}(-1)^{k}\left(\frac{f^{(k)}(b) b^{k+1}-f^{(k)}(a) a^{k+1}}{(k+1) !}\right)-\int_{a}^{b} f(x) d x\right| \\
& \leqslant \frac{1}{n !}(b-a) L_{n p}^{n}(a, b) A^{\frac{1}{q}}\left(\left|f^{(n)}(a)\right|^{q},\left|f^{(n)}(b)\right|^{q}\right) .
\end{aligned}
$$

Proof. If $\left|f^{(n)}\right|^{q}$ for $q>1$ is convex on $[a, b]$, using Lemma 2.1, the Hölder integral inequality and

$$
\left|f^{(n)}(x)\right|^{q}=\left|f^{(n)}\left(\frac{x-a}{b-a} b+\frac{b-x}{b-a} a\right)\right|^{q} \leqslant \frac{x-a}{b-a}\left|f^{(n)}(b)\right|^{q}+\frac{b-x}{b-a}\left|f^{(n)}(a)\right|^{q},
$$

we have

$$
\left|\sum_{k=0}^{n-1}(-1)^{k}\left(\frac{f^{(k)}(b) b^{k+1}-f^{(k)}(a) a^{k+1}}{(k+1) !}\right)-\int_{a}^{b} f(x) d x\right|
$$




$$
\begin{aligned}
& \leqslant \frac{1}{n !} \int_{a}^{b} x^{n}\left|f^{(n)}(x)\right| d x \\
& \leqslant \frac{1}{n !}\left(\int_{a}^{b} x^{n p} d x\right)^{\frac{1}{p}}\left(\int_{a}^{b}\left|f^{(n)}(x)\right|^{q} d x\right)^{\frac{1}{q}} \\
& \leqslant \frac{1}{n !}\left(\int_{a}^{b} x^{n p} d x\right)^{\frac{1}{p}}\left(\int_{a}^{b}\left[\frac{x-a}{b-a}\left|f^{(n)}(b)\right|^{q}+\frac{b-x}{b-a}\left|f^{(n)}(a)\right|^{q}\right] d x\right)^{\frac{1}{q}} \\
& =\frac{1}{n !}(b-a)^{\frac{1}{q}}(b-a)^{\frac{1}{p}}\left[\frac{b^{n p+1}-a^{n p+1}}{(n p+1)(b-a)}\right]^{\frac{1}{p}}\left[\frac{\left|f^{(n)}(a)\right|^{q}+\left|f^{(n)}(b)\right|^{q}}{2}\right]^{\frac{1}{q}} \\
& =\frac{1}{n !}(b-a) L_{n p}^{n}(a, b) A^{\frac{1}{q}}\left(\left|f^{(n)}(a)\right|^{q},\left|f^{(n)}(b)\right|^{q}\right) .
\end{aligned}
$$

This completes the proof of theorem.

Corollary 2.3. Under the conditions of Theorem 2.2 for $n=1$, we have the following inequality:

$$
\left|\frac{f(b) b-f(a) a}{b-a}-\frac{1}{b-a} \int_{a}^{b} f(x) d x\right| \leqslant L_{p}(a, b)\left[\frac{\left|f^{\prime}(a)\right|^{q}+\left|f^{\prime}(b)\right|^{q}}{2}\right]^{\frac{1}{q}} .
$$

Proposition 2.4. Let $\mathrm{a}, \mathrm{b} \in(0, \infty)$ with $\mathrm{a}<\mathrm{b}, \mathrm{q}>1$ and $\mathrm{m} \in(-\infty, 0] \cup[1, \infty) \backslash\{-2 \mathrm{q},-\mathrm{q}\}$, we have

$$
\mathrm{L}_{\frac{\mathfrak{m}}{q}+1}^{\frac{m}{q}+1}(a, b) \leqslant L_{p}(a, b) A^{\frac{1}{q}}\left(a^{m}, b^{m}\right) .
$$

Proof. Under the assumption of the proposition, let $f(x)=\frac{q}{m+q} x^{\frac{m}{q}+1}, x \in(0, \infty)$. Then

$$
\left|f^{\prime}(x)\right|^{q}=x^{m}
$$

is convex on $(0, \infty)$ and the result follows directly from Corollary 2.3.

Theorem 2.5. For $\mathrm{n} \in \mathbb{N}$, let $\mathrm{f}: \mathrm{I} \subseteq(0, \infty) \rightarrow \mathbb{R}$ be $\mathrm{n}$-times differentiable function on $\mathrm{I}^{\circ}$ and $\mathrm{a}, \mathrm{b} \in \mathrm{I}^{\circ}$ with $\mathrm{a}<\mathrm{b}$. If $\mathrm{f}^{(\mathrm{n})} \in \mathrm{L}[\mathrm{a}, \mathrm{b}]$ and $\left|\mathrm{f}^{(\mathrm{n})}\right|^{\mathrm{q}}$ for $\mathrm{q} \geqslant 1$ is convex on $[\mathrm{a}, \mathrm{b}]$, then the following inequality holds:

$$
\begin{aligned}
& \left|\sum_{k=0}^{n-1}(-1)^{k}\left(\frac{f^{(k)}(b) b^{k+1}-f^{(k)}(a) a^{k+1}}{(k+1) !}\right)-\int_{a}^{b} f(x) d x\right| \\
& \leqslant \frac{1}{n !}(b-a)^{1-\frac{1}{q}} L_{n p}^{n\left(\frac{q-1}{q}\right)}(a, b) \\
& \quad \times\left\{\left|f^{(n)}(b)\right|^{q}\left[L_{n+1}^{n+1}(a, b)-a L_{n}^{n}(a, b)\right]+\left|f^{(n)}(a)\right|^{q}\left[b L_{n}^{n}(a, b)-L_{n+1}^{n+1}(a, b)\right]\right\}^{\frac{1}{q}} .
\end{aligned}
$$

Proof. From Lemma 2.1 and power-mean integral inequality, we obtain

$$
\begin{aligned}
& \left|\sum_{k=0}^{n-1}(-1)^{k}\left(\frac{f^{(k)}(b) b^{k+1}-f^{(k)}(a) a^{k+1}}{(k+1) !}\right)-\int_{a}^{b} f(x) d x\right| \\
& \leqslant \frac{1}{n !} \int_{a}^{b} x^{n}\left|f^{(n)}(x)\right| d x \\
& \leqslant \frac{1}{n !}\left(\int_{a}^{b} x^{n} d x\right)^{1-\frac{1}{q}}\left(\int_{a}^{b} x^{n}\left|f^{(n)}(x)\right|^{q} d x\right)^{\frac{1}{q}}
\end{aligned}
$$




$$
\begin{aligned}
\leqslant & \frac{1}{n !}\left(\int_{a}^{b} x^{n} d x\right)^{1-\frac{1}{q}}\left(\int_{a}^{b} x^{n}\left[\frac{x-a}{b-a}\left|f^{(n)}(b)\right|^{q}+\frac{b-x}{b-a}\left|f^{(n)}(a)\right|^{q}\right] d x\right)^{\frac{1}{q}} \\
= & \frac{1}{n !}(b-a)^{1-\frac{1}{q}}\left[\frac{b^{n+1}-a^{n+1}}{(n+1)(b-a)}\right]^{1-\frac{1}{q}} \\
& \times\left\{\left|f^{(n)}(b)\right|^{q}\left[\frac{b^{n+2}-a^{n+2}}{(n+2)(b-a)}-a \frac{b^{n+1}-a^{n+1}}{(n+1)(b-a)}\right]\right. \\
& \left.+\left|f^{(n)}(a)\right|^{q}\left[b \frac{b^{n+1}-a^{n+1}}{(n+1)(b-a)}-\frac{b^{n+2}-a^{n+2}}{(n+2)(b-a)}\right]\right\}^{\frac{1}{q}} \\
= & \frac{1}{n !}(b-a)^{1-\frac{1}{q}} L_{n p}^{n\left(\frac{q}{q}\right)}(a, b) \\
& \times\left\{\left|f^{(n)}(b)\right|^{q}\left[L_{n+1}^{n+1}(a, b)-a L_{n}^{n}(a, b)\right]+\left|f^{(n)}(a)\right|^{q}\left[b L_{n}^{n}(a, b)-L_{n+1}^{n+1}(a, b)\right]\right\}^{\frac{1}{q}} .
\end{aligned}
$$

This completes the proof of theorem.

Corollary 2.6. Under the conditions of Theorem 2.5 for $n=1$, we have the following inequality:

$$
\left|\frac{f(b) b-f(a) a}{b-a}-\frac{1}{b-a} \int_{a}^{b} f(x) d x\right| \leqslant 6^{-\frac{1}{q}}\left(\frac{a+b}{2}\right)^{1-\frac{1}{q}}\left[(2 b+a)\left|f^{\prime}(b)\right|^{q}+(b+2 a)\left|f^{\prime}(a)\right|^{q}\right]^{\frac{1}{q}} .
$$

Proposition 2.7. Let $\mathrm{a}, \mathrm{b} \in(0, \infty)$ with $\mathrm{a}<\mathrm{b}, \mathrm{q}>1$ and $\mathrm{m} \in(-\infty, 0] \cup[1, \infty) \backslash\{-2 \mathrm{q},-\mathrm{q}\}$, we have

$$
L_{\frac{m}{q}+1}^{\frac{m}{q}+1}(a, b) \leqslant 3^{-\frac{1}{q}} A^{1-\frac{1}{q}}(a, b)\left[2 A\left(a^{m+1}, b^{m+1}\right)+G^{2}(a, b) A\left(a^{m-1}, b^{m-1}\right)\right]^{\frac{1}{q}} .
$$

Proof. The result follows directly from Corollary 2.6 for the function

$$
f(x)=\frac{q}{m+q} x^{\frac{m}{q}+1}, \quad x \in(0, \infty) .
$$

This completes the proof of proposition.

Corollary 2.8. Using Proposition 2.7 for $\mathrm{m}=1$, we have the following inequality:

$$
L_{\frac{1}{q}+1}^{\frac{1}{q}+1}(a, b) \leqslant 3^{-\frac{1}{q}} A^{1-\frac{1}{q}}(a, b)\left[2 A\left(a^{2}, b^{2}\right)+G^{2}(a, b)\right]^{\frac{1}{q}} .
$$

Corollary 2.9. Using Proposition 2.7 for $\mathrm{q}=1$, we have the following inequality:

$$
L_{m+1}^{m+1}(a, b) \leqslant \frac{1}{3}\left[2 A\left(a^{m+1}, b^{m+1}\right)+G^{2}(a, b) A\left(a^{m-1}, b^{m-1}\right)\right] .
$$

Corollary 2.10. Using Corollary 2.9 for $\mathrm{m}=1$, we have the following inequality:

$$
\mathrm{L}_{2}^{2}(\mathrm{a}, \mathrm{b}) \leqslant \frac{1}{3}\left[2 \mathrm{~A}\left(\mathrm{a}^{2}, \mathrm{~b}^{2}\right)+\mathrm{G}^{2}(\mathrm{a}, \mathrm{b})\right] .
$$

Corollary 2.11. Under the conditions of Theorem 2.5 for $\mathrm{q}=1$, we have the following inequality:

$$
\begin{aligned}
& \left|\sum_{k=0}^{n-1}(-1)^{k}\left(\frac{f^{(k)}(b) b^{k+1}-f^{(k)}(a) a^{k+1}}{(k+1) !}\right)-\int_{a}^{b} f(x) d x\right| \\
& \quad \leqslant \frac{1}{n !}\left\{\left|f^{(n)}(b)\right|\left[L_{n+1}^{n+1}(a, b)-a L_{n}^{n}(a, b)\right]+\left|f^{(n)}(a)\right|\left[b L_{n}^{n}(a, b)-L_{n+1}^{n+1}(a, b)\right]\right\} .
\end{aligned}
$$


Theorem 2.12. For $\mathrm{n} \in \mathbb{N}$, let $\mathrm{f}: \mathrm{I} \subseteq(0, \infty) \rightarrow \mathbb{R}$ be $\mathrm{n}$-times differentiable function on $\mathrm{I}^{\circ}$ and $\mathrm{a}, \mathrm{b} \in \mathrm{I}^{\circ}$ with $\mathrm{a}<\mathrm{b}$. If $\mathrm{f}^{(\mathrm{n})} \in \mathrm{L}[\mathrm{a}, \mathrm{b}]$ and $\left|\mathrm{f}^{(\mathrm{n})}\right|^{\mathrm{q}}$ for $\mathrm{q}>1$ is convex on $[\mathrm{a}, \mathrm{b}]$, then the following inequality holds:

$$
\begin{aligned}
& \left|\sum_{k=0}^{n-1}(-1)^{k}\left(\frac{f^{(k)}(b) b^{k+1}-f^{(k)}(a) a^{k+1}}{(k+1) !}\right)-\int_{a}^{b} f(x) d x\right| \\
& \quad \leqslant \frac{1}{n !}(b-a)^{\frac{1}{p}}\left\{\left|f^{(n)}(b)\right|^{q}\left[L_{n q+1}^{n q+1}(a, b)-a L_{n q}^{n q}(a, b)\right]+\left|f^{(n)}(a)\right|^{q}\left[b L_{n q}^{n q}(a, b)-L_{n q+1}^{n q+1}(a, b)\right]\right\}^{\frac{1}{q}} .
\end{aligned}
$$

Proof. Since $\left|f^{(n)}\right|^{q}$ for $q>1$ is convex on $[a, b]$, using Lemma 2.1 and the Hölder integral inequality, we have the following inequality:

$$
\begin{aligned}
& \left|\sum_{k=0}^{n-1}(-1)^{k}\left(\frac{f^{(k)}(b) b^{k+1}-f^{(k)}(a) a^{k+1}}{(k+1) !}\right)-\int_{a}^{b} f(x) d x\right| \\
& \leqslant \frac{1}{n !} \int_{a}^{b} 1 \cdot x^{n}\left|f^{(n)}(x)\right| d x \\
& \leqslant \frac{1}{n !}\left(\int_{a}^{b} 1^{p} d x\right)^{\frac{1}{p}}\left(\int_{a}^{b} x^{n q}\left|f^{(n)}(x)\right|^{q} d x\right)^{\frac{1}{q}} \\
& =\frac{1}{n !}\left(\int_{a}^{b} 1 d x\right)^{\frac{1}{p}}\left(\int_{a}^{b} x^{n q}\left|f^{(n)}\left(\frac{x-a}{b-a} b+\frac{b-x}{b-a} a\right)\right|^{q} d x\right)^{\frac{1}{q}} \\
& \leqslant \frac{1}{n !}\left(\int_{a}^{b} 1 d x\right)^{\frac{1}{p}}\left(\int_{a}^{b}\left[\frac{x-a}{b-a} x^{n q}\left|f^{(n)}(b)\right|^{q}+\frac{b-x}{b-a} x^{n q}\left|f^{(n)}(a)\right|^{q}\right] d x\right)^{\frac{1}{q}} \\
& =\frac{1}{n !}(b-a)^{\frac{1}{p}}\left\{\left|f^{(n)}(b)\right|^{q}\left[\frac{b^{n q+2}-a^{n q+2}}{(n q+2)(b-a)}-a \frac{b^{n q+1}-a^{n q+1}}{(n q+1)(b-a)}\right]\right. \\
& \left.+\left|f^{(n)}(a)\right|^{q}\left[b \frac{b^{n q+1}-a^{n q+1}}{(n q+1)(b-a)}-\frac{b^{n q+2}-a^{n q+2}}{(n q+2)(b-a)}\right]\right\}^{\frac{1}{q}} \\
& =\frac{1}{n !}(b-a)^{\frac{1}{p}}\left\{\left|f^{(n)}(b)\right|^{q}\left[L_{n q+1}^{n q+1}(a, b)-a L_{n q}^{n q}(a, b)\right]+\left|f^{(n)}(a)\right|^{q}\left[b L_{n q}^{n q}(a, b)-L_{n q+1}^{n q+1}(a, b)\right]\right\}^{\frac{1}{q}} .
\end{aligned}
$$

This completes the proof of theorem.

Corollary 2.13. Under the conditions of Theorem 2.12 for $n=1$, we have the following inequality:

$$
\begin{aligned}
& \left|\frac{f(b) b-f(a) a}{b-a}-\frac{1}{b-a} \int_{a}^{b} f(x) d x\right| \\
& \leqslant\left\{\frac{\left|f^{\prime}(b)\right|^{q}}{b-a}\left[L_{q+1}^{q+1}(a, b)-a L_{q}^{q}(a, b)\right]+\frac{\left|f^{\prime}(a)\right|^{q}}{b-a}\left[b L_{q}^{q}(a, b)-L_{q+1}^{q+1}(a, b)\right]\right\}^{\frac{1}{q}} .
\end{aligned}
$$

Proposition 2.14. Let $\mathrm{a}, \mathrm{b} \in(0, \infty)$ with $\mathrm{a}<\mathrm{b}, \mathrm{q}>1$ and $\mathrm{m} \in(-\infty, 0] \cup[1, \infty) \backslash\{-2 \mathrm{q},-\mathrm{q}\}$, then we have

$$
\mathrm{L}_{\frac{m}{q}+1}^{\frac{m}{q}+1}(a, b) \leqslant(b-a)^{-\frac{1}{q}}\left\{\left(b^{m}-a^{m}\right) L_{q+1}^{q+1}(a, b)-G^{2}(a, b)\left(b^{m-1}-a^{m-1}\right) L_{q}^{q}(a, b)\right\}^{\frac{1}{q}} .
$$

Proof. The result follows directly from Corollary 2.13 for the function

$$
f(x)=\frac{q}{m+q} x^{\frac{m}{q}+1}, \quad x \in(0, \infty) .
$$

This completes the proof of proposition. 
Corollary 2.15. For $m=1$ from Proposition 2.14 , we obtain the following inequality:

$$
\mathrm{L}_{\frac{1}{\mathrm{q}}+1}^{\frac{1}{\mathrm{q}}+1}(\mathrm{a}, \mathrm{b}) \leqslant\left[\mathrm{L}_{\mathrm{q}+1}^{\mathrm{q}+1}(\mathrm{a}, \mathrm{b})\right]^{\frac{1}{\mathrm{q}}}=\mathrm{L}_{\mathrm{q}+1}^{\frac{\mathrm{q}+1}{\mathrm{q}}}(\mathrm{a}, \mathrm{b}) .
$$

Theorem 2.16. For $\mathrm{n} \in \mathbb{N}$, let $\mathrm{f}: \mathrm{I} \subset(0, \infty) \rightarrow \mathbb{R}$ be $\mathrm{n}$-times differentiable function on $\mathrm{I}^{\circ}$ and $\mathrm{a}, \mathrm{b} \in \mathrm{I}^{\circ}$ with $\mathrm{a}<\mathrm{b}$. If $\mathrm{f}^{(\mathrm{n})} \in \mathrm{L}[\mathrm{a}, \mathrm{b}]$ and $\left|\mathrm{f}^{(\mathrm{n})}\right|^{\mathrm{q}}$ for $\mathrm{q}>1$ is concave on $[\mathrm{a}, \mathrm{b}]$, then the following inequality holds:

$$
\left|\sum_{k=0}^{n-1}(-1)^{k}\left(\frac{f^{(k)}(b) b^{k+1}-f^{(k)}(a) a^{k+1}}{(k+1) !}\right)-\int_{a}^{b} f(x) d x\right| \leqslant \frac{b-a}{n !} L_{n p}^{n}(a, b)\left|f^{(n)}\left(\frac{a+b}{2}\right)\right| .
$$

Proof. Since $\left|f^{(n)}\right|^{q}$ for $q>1$ is concave on $[a, b]$, with respect to Hermite-Hadamard inequality we get

$$
\int_{a}^{b}\left|f^{(n)}(x)\right|^{q} d x \leqslant(b-a)\left|f^{(n)}\left(\frac{a+b}{2}\right)\right|^{q} .
$$

Using Lemma 2.1 and the Hölder integral inequality we have

$$
\begin{aligned}
& \left|\sum_{k=0}^{n-1}(-1)^{k}\left(\frac{f^{(k)}(b) b^{k+1}-f^{(k)}(a) a^{k+1}}{(k+1) !}\right)-\int_{a}^{b} f(x) d x\right| \\
& \leqslant \frac{1}{n !} \int_{a}^{b} x^{n}\left|f^{(n)}(x)\right| d x \\
& \leqslant \frac{1}{n !}\left(\int_{a}^{b} x^{n p} d x\right)^{\frac{1}{p}}\left(\int_{a}^{b n}\left|f^{(n)}(x)\right|^{q} d x\right)^{\frac{1}{q}} \\
& \leqslant \frac{1}{n !}\left(\int_{a}^{b} x^{n p} d x\right)^{\frac{1}{p}}\left((b-a)\left|f^{(n)}\left(\frac{a+b}{2}\right)\right|^{q}\right)^{\frac{1}{q}} \\
& \quad=\frac{b-a}{n !}\left[\frac{b^{n p+1}-a^{n p+1}}{(n p+1)(b-a)}\right]^{\frac{1}{p}}\left|f^{(n)}\left(\frac{a+b}{2}\right)\right| \\
& \quad=\frac{b-a}{n !} L_{n p}^{n}(a, b)\left|f^{(n)}\left(\frac{a+b}{2}\right)\right| .
\end{aligned}
$$

This completes the proof of theorem.

Corollary 2.17. Under the conditions Theorem 2.16 for $n=1$, we have the following inequality:

$$
\left|\frac{f(b) b-f(a) a}{b-a}-\frac{1}{b-a} \int_{a}^{b} f(x) d x\right| \leqslant L_{p}(a, b)\left|f^{\prime}\left(\frac{a+b}{2}\right)\right| .
$$

Proposition 2.18. Let $\mathrm{a}, \mathrm{b} \in(0, \infty)$ with $\mathrm{a}<\mathrm{b}, \mathrm{q}>1$ and $\mathrm{m} \in[0,1]$, then we have

$$
\mathrm{L}_{\frac{\mathrm{m}}{q}+1}^{\frac{m}{q}+1}(a, b) \leqslant L_{p}(a, b) A^{\frac{m}{q}}(a, b) .
$$

Proof. Under the assumption of the proposition, let $f(x)=\frac{q}{m+q} x^{\frac{m}{q}+1}, x \in(0, \infty)$. Then

$$
\left|f^{\prime}(x)\right|^{q}=x^{m}
$$

is concave on $(0, \infty)$ and the result follows directly from Corollary 2.17. 


\section{References}

[1] M. Alomari, M. Darus, S. S. Dragomir, New inequalities of Hermite-Hadamard type for functions whose second derivatives absolute values are quasi-convex, Tamkang J. Math., 41 (2010), 353-359. 1

[2] S.-P. Bai, S.-H. Wang, F. Qi, Some Hermite-Hadamard type inequalities for $\mathrm{n}$-time differentiable $(\alpha, \mathrm{m})$-convex functions, J. Inequal. Appl., 2012 (2012), 11 pages. 1

[3] P. Cerone, S. S. Dragomir, J. Roumeliotis, Some Ostrowski type inequalities for n-time differentiable mappings and applications, Demonstratio Math., 32 (1999), 697-712. 1

[4] P. Cerone, S. S. Dragomir, J. Roumeliotis, J. Sunde, A new generalization of the trapezoid formula for n-time differentiable mappings and applications, Demonstratio Math., 33 (2000), 719-736. 1

[5] S. S. Dragomir, C. E. M. Pearce, Selected Topics on Hermite-Hadamard Inequalities and Applications, Victoria University, Australia, (2000). 1

[6] D.-Y. Hwang, Some Inequalities for n-time Differentiable Mappings and Applications, Kyungpook Math. J., 43 (2003), 335-343. 1

[7] İ. İşcan, Hermite-Hadamard type inequalities for harmonically convex functions, Hacet. J. Math. Stat., 43 (2014), $935-942$. 1

[8] İ. İşcan, Ostrowski type inequalities for p-convex functions, New Trends Math. Sci., 4 (2016), 140-150.

[9] İ. İşcan, M. Kunt, Hermite-Hadamard-Fejer type inequalities for harmonically quasi-convex functions via fractional integrals, Kyungpook Math. J., 56 (2016), 845-859.

[10] İ. İşcan, M. Kunt, Hermite-Hadamard-Fejer type inequalities for quasi-geometrically convex functions via fractional integrals, J. Math., 2016 (2016), 7 pages.

[11] İ. İşcan, S. Turhan, Generalized Hermite-Hadamard-Fejer type inequalities for GA-convex functions via Fractional integral, Moroccan J. Pure Appl. Anal., 2 (2016), 34-46. 1

[12] İ. İşcan, S. Turhan, S. Maden, Some Hermite-Hadamard-Fejer type inequalities for Harmonically convex functions via Fractional Integral, New Trends Math. Sci., 4 (2016), 1-10. 1

[13] W.-D. Jiang, D.-W. Niu, Y. Hua, F. Qi, Generalizations of Hermite-Hadamard inequality to n-time differentiable function which are s-convex in the second sense, Analysis (Munich), 32 (2012), 209-220. 1

[14] U. S. Kirmaci, M. K. Bakula, M. E. Özdemir, J. Pečarić, Hadamard-type inequalities for s-convex functions, Appl. Math. Comp., 193 (2007), 26-35. 1

[15] M. E. Özdemir, Ç. Yıld1z, New Inequalities for Hermite-Hadamard and Simpson Type with Applications, Tamkang J. Math., 44 (2013), 209-216. 1

[16] J. E. Pečarić, F. Porschan, Y. L. Tong, Convex Functions, Partial Orderings, and Statistical Applications, Academic Press, Boston, (1992). 1

[17] S.-H. Wang, B.-Y. Xi, F. Qi, Some new inequalities of Hermite-Hadamard type for n-time differentiable functions which are m-convex, Analysis (Munich), 32 (2012), 247-262. 1

[18] B.-Y. Xi, F. Qi, Some integral inequalities of Hermite-Hadamard type for convex functions with applications to means, J. Funct. Spaces Appl., 2012 (2012), 14 pages. 1

[19] Ç. Yıldız, New inequalities of the Hermite-Hadamard type for n-time differentiable functions which are quasiconvex, J. Math. Inequal., 10 (2016), 703-711. 1 